\title{
Yang-Baxter Maps from the Discrete BKP Equation ${ }^{\star}$
}

\author{
Saburo KAKEI ${ }^{\dagger}$, Jonathan J.C. NIMMO ${ }^{\ddagger}$ and Ralph WILLOX \\ $\dagger$ Department of Mathematics, College of Science, Rikkyo University, \\ 3-34-1 Nishi-Ikebukuro, Toshima-ku, Tokyo 171-8501, Japan \\ E-mail:kakei@rikkyo.ac.jp \\ URL: http://www.rkmath.rikkyo.ac.jp/ kakei/index_e.html \\ $\ddagger$ Department of Mathematics, University of Glasgow, Glasgow G12 8QQ, UK \\ E-mail: j.nimmo@maths.gla.ac.uk \\ URL: http://www.maths.gla.ac.uk/ jjcn/ \\ $\S$ Graduate School of Mathematical Sciences, The University of Tokyo, \\ 3-8-1 Komaba, Meguro-ku, Tokyo 153-8914, Japan \\ E-mail:willox@ms.u-tokyo.ac.jp
}

Received November 13, 2009, in final form March 19, 2010; Published online March 31, 2010

doi:10.3842/SIGMA.2010.028

\begin{abstract}
We construct rational and piecewise-linear Yang-Baxter maps for a general $N$-reduction of the discrete BKP equation.
\end{abstract}

Key words: Yang-Baxter map; discrete BKP equation

2010 Mathematics Subject Classification: 37K10; 37K60; 39A10; 39A14

\section{Introduction}

Let $R$ be a map $R: \mathbb{C}^{2} \rightarrow \mathbb{C}^{2}$ and let $R_{i j}: \mathbb{C}^{n} \rightarrow \mathbb{C}^{n}$ be the map that acts as $R$ on the $i$-th and $j$-th factors in the direct product $\mathbb{C} \times \cdots \times \mathbb{C}$ and identically on the others. A map $R$ is called a Yang-Baxter map if it satisfies the Yang-Baxter equation (with parameters) [23, 24],

$$
R_{12}\left(\lambda_{1}, \lambda_{2}\right) R_{13}\left(\lambda_{1}, \lambda_{3}\right) R_{23}\left(\lambda_{2}, \lambda_{3}\right)=R_{23}\left(\lambda_{2}, \lambda_{3}\right) R_{13}\left(\lambda_{1}, \lambda_{3}\right) R_{12}\left(\lambda_{1}, \lambda_{2}\right) .
$$

An important example of a Yang-Baxter map can be derived from the 3D-consistency condition for the discrete potential KdV equation [17]:

$$
R(\kappa, \mu):(u, v) \mapsto\left(v p, \frac{u}{p}\right), \quad p=\frac{1+\kappa u v}{1+\mu u v},
$$

which is the $H_{\mathrm{III}}^{B}$ map in the classification of quadrirational Yang-Baxter maps found in [16] (which is in fact equivalent to the $F_{\text {III }}$ map in the Adler-Bobenko-Suris classification [1]). An important property of the map (2) is that it is related to a geometric crystal of $A_{1}^{(1)}$-type [5]. Indeed, if we set

$$
u=e^{U / \epsilon}, \quad v=e^{V / \epsilon}, \quad p=e^{P / \epsilon}, \quad \kappa=e^{-K / \epsilon}, \quad \mu=e^{-M / \epsilon},
$$

and take the ultra-discrete limit (henceforth "ud-limit") $\epsilon \rightarrow+0$ [22], we obtain a piecewiselinear Yang-Baxter map,

$$
(U, V) \mapsto(V+P, U-P), \quad P=\max (0, U+V-K)-\max (0, U+V-M),
$$

* This paper is a contribution to the Proceedings of the Workshop "Geometric Aspects of Discrete and UltraDiscrete Integrable Systems" (March 30 - April 3, 2009, University of Glasgow, UK). The full collection is available at http://www.emis.de/journals/SIGMA/GADUDIS2009.html 
where we have used the formula

$$
\lim _{\epsilon \rightarrow+0} \epsilon \log \left(e^{A / \epsilon}+e^{B / \epsilon}\right)=\max (A, B), \quad A, B \in \mathbb{R} .
$$

It can be verified that (3) coincides with the combinatorial $R$-matrix of $A_{1}^{(1)}$-type [6], and is in fact equivalent to the evolution rule of a soliton cellular automata (a box-ball system "with a carrier") [20]. Furthermore, if we choose $\kappa=0$, the map (2) coincides with Hirota's discrete $\mathrm{KdV}$ equation [8] and is therefore related to the Takahashi-Satsuma soliton cellular automata [21] as discussed in [11].

In a previous paper [11], we showed how several Yang-Baxter maps can be directly obtained from the discrete KP hierarchy. The aim of the present paper is to investigate the BKP counterpart of this construction and of the map (2) in particular.

\section{Hirota-Miwa equation and Harrison-type map}

In this section, we briefly describe a non-autonomous version of the method that enabled us to obtain the map (2) from the Hirota-Miwa equation,

$$
\begin{aligned}
& (b-c) \tau(\ell+1, m, n) \tau(\ell, m+1, n+1)+(c-a) \tau(\ell, m+1, n) \tau(\ell+1, m, n+1) \\
& +(a-b) \tau(\ell, m, n+1) \tau(\ell+1, m+1, n)=0,
\end{aligned}
$$

which was introduced by Hirota [9]. The connection between (5) and the the $A$-type KP hierarchy was clarified by Miwa [13]. A non-autonomous version of the Hirota-Miwa equation,

$$
\begin{gathered}
\{b(m)-c(n)\} \tau(100) \tau(011)+\{c(n)-a(\ell)\} \tau(010) \tau(101) \\
+\{a(\ell)-b(m)\} \tau(001) \tau(110)=0
\end{gathered}
$$

was studied in $[25,26]$. Here we have used the abbreviations, $\tau(100)=\tau(\ell+1, m, n), \tau(101)=$ $\tau(\ell+1, m, n+1)$, etc. The non-autonomous Hirota-Miwa (naHM) equation (6) arises as the compatibility condition for the linear system (cf. [3, 14] for autonomous case),

$$
\begin{aligned}
& \psi(010)=\frac{a(\ell)}{b(m)} \psi(100)+\left\{1-\frac{a(\ell)}{b(m)}\right\} u(000) \psi(000), \\
& \psi(001)=\frac{a(\ell)}{c(n)} \psi(100)+\left\{1-\frac{a(\ell)}{c(n)}\right\} v(000) \psi(000),
\end{aligned}
$$

where $u(\ell, m, n), v(\ell, m, n)$ are defined as

$$
u(000)=\frac{\tau(000) \tau(110)}{\tau(100) \tau(010)}, \quad v(000)=\frac{\tau(000) \tau(101)}{\tau(100) \tau(001)} .
$$

We now impose the following 2-reduction condition with respect to the variable $\ell$;

$$
\begin{aligned}
& u(\ell+2, m, n)=u(\ell, m, n), \quad v(\ell+2, m, n)=v(\ell, m, n), \\
& \psi(\ell+2, m, n)=\lambda \psi(\ell, m, n), \quad a(\ell+2)=a(\ell) \quad \forall \ell \in \mathbb{Z} .
\end{aligned}
$$

We furthermore require that the evolution in the remaining lattice directions is autonomous:

$$
b(m)=b, \quad c(n)=c \quad \forall m, n \in \mathbb{Z} .
$$

Then the linear system ( 7$)$ is reduced to the $2 \times 2$ system

$$
\left[\begin{array}{l}
\psi_{0}(m+1, n) \\
\psi_{1}(m+1, n)
\end{array}\right]=\mathcal{U}(m, n)\left[\begin{array}{l}
\psi_{0}(m, n) \\
\psi_{1}(m, n)
\end{array}\right], \quad\left[\begin{array}{l}
\psi_{0}(m, n+1) \\
\psi_{1}(m, n+1)
\end{array}\right]=\mathcal{V}(m, n)\left[\begin{array}{l}
\psi_{0}(m, n) \\
\psi_{1}(m, n)
\end{array}\right]
$$


where $\psi_{i}(m, n)=\psi(i, m, n)(i=0,1)$ and the coefficient matrices $\mathcal{U}(m, n), \mathcal{V}(m, n)$ are given by

$$
\begin{aligned}
& \mathcal{U}(m, n)=\left[\begin{array}{cc}
\{1-a(0) / b\} u_{0}(m, n) & a(0) / b \\
\lambda a(1) / b & \{1-a(1) / b\} u_{1}(m, n)
\end{array}\right] \\
& \mathcal{V}(m, n)=\left[\begin{array}{cc}
\{1-a(0) / c\} v_{0}(m, n) & a(0) / c \\
\lambda a(1) / c & \{1-a(1) / c\} v_{1}(m, n)
\end{array}\right], \\
& u_{\ell}(m, n)=u(\ell, m, n), \quad v_{\ell}(m, n)=v(\ell, m, n) .
\end{aligned}
$$

We remark that $u_{\ell}(m, n), v_{\ell}(m, n)(\ell=0,1)$ obey the constraints

$$
u_{0}(m, n) u_{1}(m, n)=v_{0}(m, n) v_{1}(m, n)=1,
$$

under the conditions (9), (10).

The discrete Lax equation

$$
\mathcal{U}(m, n+1) \mathcal{V}(m, n)=\mathcal{V}(m+1, n) \mathcal{U}(m, n),
$$

follows from the compatibility condition for (11) and gives

$$
\begin{aligned}
& u_{\ell}(m, n) v_{\ell}(m+1, n)=u_{\ell}(m, n+1) v_{\ell}(m, n) \\
& \{a(\ell)-b\} u_{\ell}(m, n+1)-\{a(\ell)-c\} v_{\ell}(m+1, n) \\
& \quad=\{a(\ell+1)-b\} u_{\ell+1}(m, n)-\{a(\ell+1)-c\} v_{\ell+1}(m, n),
\end{aligned}
$$

for $\ell=0,1$. Using (12) to eliminate the $u_{1}, v_{1}$ variables, one can solve (14) and (15) for $u_{0}(m, n+1)$ and $v_{0}(m+1, n)$, to obtain the 2-reduced non-autonomous discrete KP (na-dKP) equation,

$$
\begin{aligned}
& u_{0}(m, n+1)=\frac{\tilde{F}(m, n)}{v_{0}(m, n)}, \quad v_{0}(m+1, n)=\frac{\tilde{F}(m, n)}{u_{0}(m, n)}, \\
& \tilde{F}(m, n)=\frac{\{a(1)-b\} v_{0}(m, n)-\{a(1)-c\} u_{0}(m, n)}{\{a(0)-b\} u_{0}(m, n)-\{a(0)-c\} v_{0}(m, n)} .
\end{aligned}
$$

Alternatively, we can use (12), (14) and (15) to express $u_{0}(m, n+1), v_{0}(m, n)$ in terms of $u_{0}(m, n), v_{0}(m+1, n)$ :

$$
\begin{aligned}
& u_{0}(m, n+1)=v_{0}(m+1, n) F(m, n), \quad v_{0}(m, n)=\frac{u_{0}(m, n)}{F(m, n)}, \\
& F(m, n)=\frac{\{a(1)-b\}+\{a(0)-c\} u_{0}(m, n) v_{0}(m+1, n)}{\{a(1)-c\}+\{a(0)-b\} u_{0}(m, n) v_{0}(m+1, n)} .
\end{aligned}
$$

Motivated by (16) and (17), we define the following birational maps $\tilde{R}(b, c), R(b, c)$ :

$$
\begin{aligned}
\tilde{R}(b, c):(u, v) \mapsto\left(\frac{\tilde{F}}{v}, \frac{\tilde{F}}{u}\right), & \tilde{F}=\frac{\{a(1)-b\} v-\{a(1)-c\} u}{\{a(0)-b\} u-\{a(0)-c\} v}, \\
R(b, c):(u, v) \mapsto\left(v F, \frac{u}{F}\right), & F=\frac{\{a(1)-b\}+\{a(0)-c\} u v}{\{a(1)-c\}+\{a(0)-b\} u v}
\end{aligned}
$$

From now on we shall refer to the map $\tilde{R}(b, c)$ as the "solitonic" map, since $\tilde{R}$ is nothing but the evolution rule for the discrete soliton equation (16). On the other hand, if we rescale as $u \mapsto\{a(1)-b\} u, v \mapsto\{a(1)-c\} v$, the map $R(b, c)$ coincides with the map (2) with $\kappa=$ $\{a(0)-c\}\{a(1)-c\}, \mu=\{a(0)-b\}\{a(1)-b\}$. Hence $R(b, c)$ is a genuine Yang-Baxter map, 
i.e. it satisfies the Yang-Baxter equation with parameters (1). As discussed in [18], this is a direct consequence of the 3D-consistency of the discrete Lax equation (13). The solitonic map $\tilde{R}(b, c)$ satisfies a similar but slightly different relation,

$$
\tilde{R}_{12}(b, c) \tilde{R}_{13}(b, d) R_{23}(c, d)=R_{23}(c, d) \tilde{R}_{13}(b, d) \tilde{R}_{12}(b, c),
$$

which is also obtained from the 3D-consistency of the Lax pair.

Remark. Here we use the notation $\tilde{R}$ for "solitonic" maps and $R$ for Yang-Baxter maps, whereas the opposite notation was used in [11].

If we impose the $N$-reduction conditions,

$$
\begin{aligned}
& u(\ell+N, m, n)=u(\ell, m, n), \quad v(\ell+N, m, n)=v(\ell, m, n), \\
& \psi(\ell+N, m, n)=\lambda \psi(\ell, m, n), \quad a(\ell+N)=a(\ell) \quad \forall \ell \in \mathbb{Z}, \\
& b(m)=b, \quad c(n)=c \quad \forall m, n \in \mathbb{Z},
\end{aligned}
$$

instead of the 2-reduction conditions (9), (10), the resulting Yang-Baxter map are related to a geometric crystal of $A_{N-1}^{(1)}$-type [5]. Its ud-limit coincides with a combinatorial $R$-matrix of $A_{N-1}^{(1)}$-type and hence with the evolution rule for $A_{N-1}^{(1)}$-soliton cellular automata [6].

\section{From the Miwa equation to Yang-Baxter maps}

In [13], Miwa introduced a discrete analogue of the bilinear BKP equation,

$$
\begin{aligned}
& (a+b)(a+c)(b-c) \tau(100) \tau(011)+(b+c)(b+a)(c-a) \tau(010) \tau(101) \\
& \quad+(c+a)(c+b)(a-b) \tau(001) \tau(110)+(a-b)(b-c)(c-a) \tau(111) \tau(000)=0 .
\end{aligned}
$$

It is straightforward to generalize (21) to the non-autonomous case:

$$
\begin{aligned}
\{a(\ell) & +b(m)\}\{a(\ell)+c(n)\}\{b(m)-c(n)\} \tau(100) \tau(011) \\
& +\{b(m)+c(n)\}\{b(m)+a(\ell)\}\{c(n)-a(\ell)\} \tau(010) \tau(101) \\
& +\{c(n)+a(\ell)\}\{c(n)+b(m)\}\{a(\ell)-b(m)\} \tau(001) \tau(110) \\
& +\{a(\ell)-b(m)\}\{b(m)-c(n)\}\{c(n)-a(\ell)\} \tau(111) \tau(000)=0 .
\end{aligned}
$$

The non-autonomous Miwa (naM) equation (22) arises as the compatibility condition for the linear system (cf. $[4,15]$ for the autonomous case),

$$
\begin{aligned}
& \{a(\ell)+b(m)\} \psi(010)-\{a(\ell)-b(m)\} u(000) \psi(110) \\
& \quad+\{a(\ell)-b(m)\} u(000) \psi(000)-\{b(m)+a(\ell)\} \psi(100)=0 \\
& \{a(\ell)+c(n)\} \psi(001)-\{a(\ell)-c(n)\} v(000) \psi(101) \\
& \quad+\{a(\ell)-c(n)\} u(000) \psi(000)-\{c(n)+a(\ell)\} \psi(100)=0
\end{aligned}
$$

where $u(\ell, m, n), v(\ell, m, n)$ are defined as in (8).

We now impose the $N$-reduction condition (20) to obtain the discrete linear equations

$$
\begin{aligned}
& \mathcal{U}_{1}(m, n)\left[\begin{array}{c}
\psi_{0}(m+1, n) \\
\vdots \\
\psi_{N-1}(m+1, n)
\end{array}\right]=\mathcal{U}_{2}(m, n)\left[\begin{array}{c}
\psi_{0}(m, n) \\
\vdots \\
\psi_{N-1}(m, n)
\end{array}\right], \\
& \mathcal{V}_{1}(m, n)\left[\begin{array}{c}
\psi_{0}(m, n+1) \\
\vdots \\
\psi_{N-1}(m, n+1)
\end{array}\right]=\mathcal{V}_{2}(m, n)\left[\begin{array}{c}
\psi_{0}(m, n) \\
\vdots \\
\psi_{N-1}(m, n)
\end{array}\right],
\end{aligned}
$$


where $\psi_{i}(m, n)=\psi(i, m, n)(i=0,1)$ and the coefficient matrices $\mathcal{U}_{i}(m, n), \mathcal{V}_{i}(m, n)(i=1,2)$ are given by

$$
\begin{aligned}
& \mathcal{U}_{1}=\mathcal{I}+\mathcal{U}^{(0)} \Lambda, \quad \mathcal{U}_{2}=\mathcal{U}^{(0)}+\Lambda, \quad \mathcal{U}^{(0)}=\operatorname{diag}\left[\beta_{0} u_{0}, \ldots, \beta_{N-1} u_{N-1}\right], \\
& \mathcal{V}_{1}=\mathcal{I}+\mathcal{V}^{(0)} \Lambda, \quad \mathcal{V}_{2}=\mathcal{V}^{(0)}+\Lambda, \quad \mathcal{V}^{(0)}=\operatorname{diag}\left[\gamma_{0} v_{0}, \ldots, \gamma_{N-1} v_{N-1}\right], \\
& u_{\ell}(m, n)=u(\ell, m, n), \quad v_{\ell}(m, n)=v(\ell, m, n) \text {, } \\
& \Lambda=\left[\begin{array}{cccc}
0 & 1 & & \\
& 0 & \ddots & \\
& & \ddots & 1 \\
\lambda & & & 0
\end{array}\right], \quad \beta_{\ell}=\frac{b-a(\ell)}{b+a(\ell)}, \quad \gamma_{\ell}=\frac{c-a(\ell)}{c+a(\ell)},
\end{aligned}
$$

where $\mathcal{I}$ is the identity matrix. We remark that $u_{\ell}(m, n), v_{\ell}(m, n)(\ell=0,1, \ldots, N-1)$ obey the constraints

$$
u_{0}(m, n) \cdots u_{N-1}(m, n)=v_{0}(m, n) \cdots v_{N-1}(m, n)=1,
$$

under the conditions (20). The compatibility condition of (23) and (24) is

$$
\begin{aligned}
& \mathcal{U}_{1}(m, n+1)^{-1} \mathcal{U}_{2}(m, n+1) \mathcal{V}_{1}(m, n)^{-1} \mathcal{V}_{2}(m, n) \\
& \quad=\mathcal{V}_{1}(m+1, n)^{-1} \mathcal{V}_{2}(m+1, n) \mathcal{U}_{1}(m, n)^{-1} \mathcal{U}_{2}(m, n) .
\end{aligned}
$$

We remark that $\operatorname{det} \mathcal{U}_{1}=\operatorname{det} \mathcal{V}_{1}=1+(-1)^{N-1} \lambda \prod_{j=0}^{N-1} \beta_{j}$ and hence $\mathcal{U}_{1}, \mathcal{V}_{1}$ are invertible.

To write down the Yang-Baxter map and the solitonic map associated with the discrete Lax equation (27), we use the abbreviations, $u_{j}:=u_{j}(0,0), v_{j}:=v_{j}(0,0), \bar{u}_{j}:=u_{j}(0,1)$, $\bar{v}_{j}:=v_{j}(1,0), \boldsymbol{u}=\left(u_{0}, \ldots, u_{N-1}\right), \boldsymbol{v}=\left(v_{0}, \ldots, v_{N-1}\right), \overline{\boldsymbol{u}}=\left(\bar{u}_{0}, \ldots, \bar{u}_{N-1}\right), \overline{\boldsymbol{v}}=\left(\bar{v}_{0}, \ldots, \bar{v}_{N-1}\right)$. Using these abbreviations, we have the following discrete evolution equations:

$$
\begin{aligned}
& \bar{u}_{j}=u_{j} \frac{f_{j+1}(\boldsymbol{u}, \boldsymbol{v})}{f_{j}(\boldsymbol{u}, \boldsymbol{v})}, \quad \bar{v}_{j}=v_{j} \frac{f_{j+1}(\boldsymbol{u}, \boldsymbol{v})}{f_{j}(\boldsymbol{u}, \boldsymbol{v})}, \quad j=0,1, \ldots, N-1, \\
& f_{j}(\boldsymbol{u}, \boldsymbol{v})=\sum_{k=0}^{N-1} \prod_{l=0}^{k-1} \beta_{j+l} \gamma_{j+l} u_{j+l} v_{j+l}\left(\beta_{j+k} u_{j+k}-\gamma_{j+k} v_{j+k}\right) .
\end{aligned}
$$

Here and in what follows, the subscripts are considered as elements of $\mathbb{Z} / N \mathbb{Z}$.

One can show that the discrete Lax equation (27) is equivalent to

$$
\begin{aligned}
& \bar{u}_{j}=u_{j} \frac{g_{j}(\boldsymbol{u}, \overline{\boldsymbol{v}})}{g_{j+1}(\boldsymbol{u}, \overline{\boldsymbol{v}})}, \quad v_{j}=\bar{v}_{j} \frac{g_{j+1}(\boldsymbol{u}, \overline{\boldsymbol{v}})}{g_{j}(\boldsymbol{u}, \overline{\boldsymbol{v}})}, \quad j=0,1, \ldots, N-1, \\
& g_{j}(\boldsymbol{u}, \overline{\boldsymbol{v}})=\sum_{k=0}^{N-1}\left\{\prod_{l=0}^{k-1} \gamma_{j+l} \bar{v}_{j+l} \cdot\left(\beta_{j+k} \gamma_{j+k} u_{j+k} \bar{v}_{j+k}-1\right) \cdot \prod_{l=k+1}^{N-1} \beta_{j+l} u_{j+l}\right\} .
\end{aligned}
$$

We will give a derivation of the equations (28), (29) in Appendix A.

In analogy with (18) we shall refer to the equations (28) as a solitonic map:

$$
\begin{array}{cccc}
\tilde{R}(\boldsymbol{\beta}, \gamma): & : \mathbb{C}^{N} \times \mathbb{C}^{N} & \rightarrow & \mathbb{C}^{N} \times \mathbb{C}^{N} \\
\Psi & & \Psi \\
(\boldsymbol{u}, \boldsymbol{v}) & \mapsto & (\overline{\boldsymbol{u}}, \overline{\boldsymbol{v}})
\end{array} .
$$

The corresponding Yang-Baxter map is obtained from (29):

$$
\begin{array}{cccc}
R(\boldsymbol{\beta}, \boldsymbol{\gamma}): & \mathbb{C}^{N} \times \mathbb{C}^{N} & \rightarrow & \mathbb{C}^{N} \times \mathbb{C}^{N} \\
\Psi & & \Psi \\
& (\boldsymbol{u}, \overline{\boldsymbol{v}}) & \mapsto & (\overline{\boldsymbol{u}}, \boldsymbol{v})
\end{array} .
$$




$$
\begin{gathered}
\left(U_{0}, U_{1}\right) \\
R:\left(\bar{V}_{0}, \bar{V}_{1}\right)\left(V_{0}, V_{1}\right) \\
\left(\bar{U}_{0}, \bar{U}_{1}\right)
\end{gathered}
$$

Figure 1. Graphical representation.

The birational map (31) satisfies the Yang-Baxter equation (1), whereas when considered together, the Yang-Baxter map (31) and the BKP solitonic map (30) satisfy (19). We remark again that these "Yang-Baxter" properties are mere consequences of the 3D consistency of the discrete Lax equation (27).

Next we consider the ud-limit of the Yang-Baxter map (29), (31). To do this, we replace the parameters $\beta$ and $\gamma$ by

$$
\beta_{j} \mapsto \sqrt{-1} \beta_{j}, \quad \gamma_{j} \mapsto \sqrt{-1} \gamma_{j}, \quad j=0,1, \ldots, N-1,
$$

and introduce new independent variables $U_{j}, V_{j}, \bar{U}_{j}, \bar{V}_{j}$ as defined in

$$
e^{U_{j} / \epsilon}=\beta_{j} u_{j}, \quad e^{V_{j} / \epsilon}=\gamma_{j} v_{j} \quad e^{\bar{U}_{j} / \epsilon}=\beta_{j} \bar{u}_{j}, \quad e^{\bar{V}_{j} / \epsilon}=\gamma_{j} \bar{v}_{j}, \quad j=0,1, \ldots, N-1 .
$$

It follows from (26) that $\sum_{j=0}^{N-1} U_{j}=\sum_{j=0}^{N-1} \bar{U}_{j}$ and $\sum_{j=0}^{N-1} V_{j}=\sum_{j=0}^{N-1} \bar{V}_{j}$ are independent of $m$ and $n$. Then taking the ud-limit of (29) gives

$$
\begin{aligned}
& \bar{U}_{j}=U_{j}+G_{j}(\boldsymbol{U}, \overline{\boldsymbol{V}})-G_{j+1}(\boldsymbol{U}, \overline{\boldsymbol{V}}), \\
& V_{j}=\bar{V}_{j}+G_{j+1}(\boldsymbol{U}, \overline{\boldsymbol{V}})-G_{j}(\boldsymbol{U}, \overline{\boldsymbol{V}}), \quad j=0,1, \ldots, N-1, \\
& G_{j}(\boldsymbol{U}, \overline{\boldsymbol{V}})=\max _{0 \leq k<N}\left(\sum_{l=0}^{k-1} \bar{V}_{j+1}+\max \left(0, U_{j+k}+\bar{V}_{j+k}\right)+\sum_{l=k+1}^{N-1} U_{j+l}\right), \\
& \boldsymbol{U}=\left(U_{0}, \ldots, U_{N-1}\right), \quad \boldsymbol{V}=\left(V_{0}, \ldots, V_{N-1}\right), \\
& \overline{\boldsymbol{U}}=\left(\bar{U}_{0}, \ldots, \bar{U}_{N-1}\right), \quad \overline{\boldsymbol{V}}=\left(\bar{V}_{0}, \ldots, \bar{V}_{N-1}\right),
\end{aligned}
$$

which defines a piecewise-linear Yang-Baxter map $R^{(\mathrm{ud})}$ :

$$
\begin{array}{cccc}
R^{(\mathrm{ud})}: & \mathbb{R}^{N} & \rightarrow & \mathbb{R}^{N} \\
& \Psi & & \Psi \\
& (\boldsymbol{U}, \overline{\boldsymbol{V}}) & \mapsto & (\overline{\boldsymbol{U}}, \boldsymbol{V})
\end{array}
$$

We remark that if we impose the condition $U_{j}, \bar{V}_{j} \geq 0(j=0,1, \ldots, N-1)$, the Yang-Baxter map $R^{(\text {ud) }}$ coincides with the combinatorial $R$-matrix of type $A_{N-1}^{(1)}$ (see (2.12) in [19]).

However, the piecewise-linear map (32) also admits soliton-type solutions in the general case. To describe these solitons, we use the notation of Fig. 1. Figs. 2 and 3 show soliton-type phenomena in the 3 -reduced case, where we have set $U_{0}+U_{1}+U_{2}=\bar{U}_{0}+\bar{U}_{1}+\bar{U}_{2}=1$ and $\bar{V}_{0}+\bar{V}_{1}+\bar{V}_{2}=V_{0}+V_{1}+V_{2}=0$. The evolution shown in Fig. 3 is reminiscent of the interactions of solitons with stationary solutions that have been studied by Hirota in case of the ultra-discrete limit of the discrete Sawada-Kotera equation [10].

\section{Concluding remarks}

In this article, we have constructed rational and piecewise-linear Yang-Baxter maps from the $N$-reduction of the discrete BKP equation. It should be remarked that the $4 \mathrm{D}$ consistency property [12] of the (non-reduced) BKP equation was observed in [1]. 


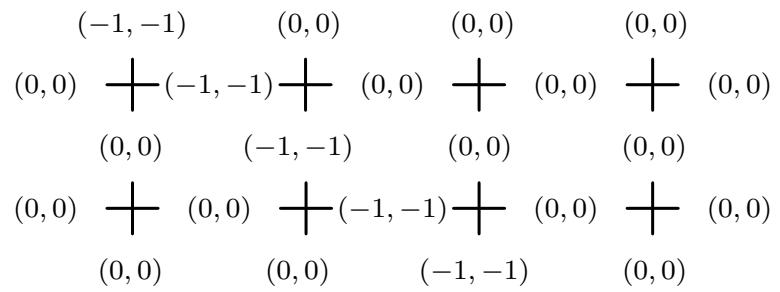

Figure 2. 1-soliton propagation.

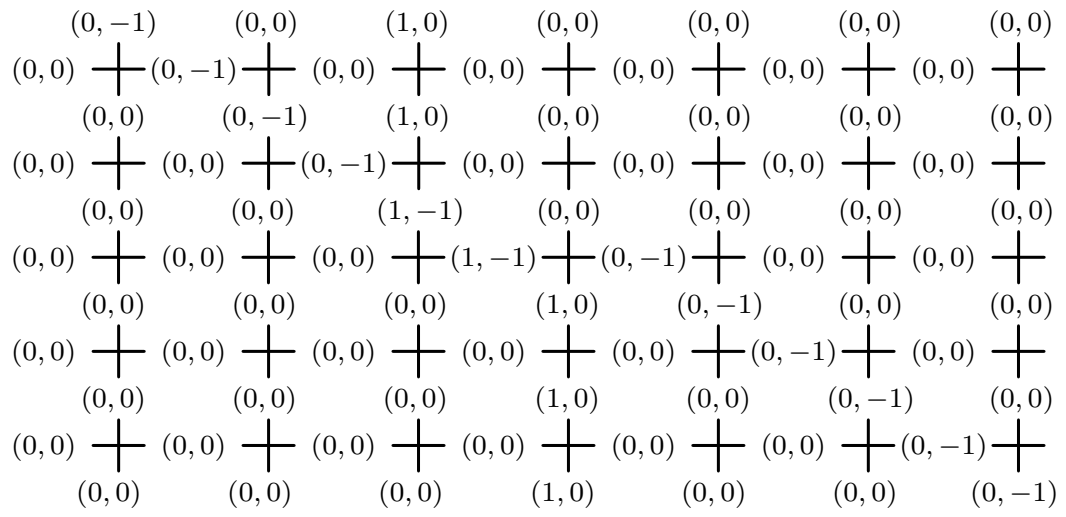

Figure 3. 2-soliton interaction.

From the Lie-algebraic viewpoint, the $(2 n+1)$-reduced BKP equation has the symmetry of the affine Lie algebra of $A_{2 n}^{(2)}$-type and the (2n)-reduced one has that of $D_{n+1}^{(2)}$ [2]. It is to be expected that the reduced ultra-discrete BKP system (32) is somehow related to crystal bases of the above types. However, in the 3-reduced case, our piecewise-linear map (32) seems to be different from the combinatorial $R$-matrix of $A_{2}^{(2)}$-type, discussed explicitly in [7], as (32) has a rotational symmetry which the $A_{2}^{(2)}$-type map in [7] does not possess. The exact relationship between the $N$-reduced discrete BKP equation and $A_{2}^{(2)}$-type crystal bases needs further clarification.

\section{A Derivation of the $N$-reduced BKP equation and related Yang-Baxter map}

Here we give a derivation of the $N$-reduced BKP equation (28) and the related Yang-Baxter map (29).

In the case $N=2$, the nonlinear equations associated with the discrete Lax equation (27) are the same as in the 2-reduced $A$-case, i.e., those in Section 2 (as is to be expected for Lie-algebraic reasons). The first non-trivial example is the 3 -reduced case and hereafter we assume $N \geq 3$.

If $\beta_{j} \neq 0, \gamma_{j} \neq 0(j=0,1, \ldots, N-1)$ in $(25)$, we can set $\beta_{j}=\gamma_{j}=1(j=0,1, \ldots, N-1)$ by rescaling the variables $\left\{u_{j}\right\},\left\{v_{j}\right\}$. In this appendix, we choose $\beta_{j}=\gamma_{j}=1$.

We first remark that the inverse of $\mathcal{U}_{1}$ of $(25)$ is of the form,

$$
\mathcal{U}_{1}^{-1}=\left\{1+(-1)^{N-1} \lambda \prod_{i=0}^{N-1} \beta_{i}\right\}^{-1}\left\{\mathcal{I}+\sum_{j=1}^{N-1}(-1)^{j}\left(\prod_{k=0}^{j-1} \mathcal{U}^{(k)}\right) \Lambda^{j}\right\}
$$

where the matrices

$$
\mathcal{U}^{(k)}=\operatorname{diag}\left[u_{k}, u_{k+1}, \ldots, u_{k+N-1}\right], \quad k=0,1, \ldots, N-1,
$$


have the properties,

$$
\mathcal{U}^{(k+N)}=\mathcal{U}^{(k)}, \quad \Lambda \mathcal{U}^{(k)}=\mathcal{U}^{(k+1)} \Lambda, \quad k=0,1, \ldots, N-1 .
$$

We then introduce a degree on $\operatorname{Mat}(N)$, the space of $N \times N$ matrices:

$$
\forall X \in \operatorname{Mat}(N) \quad \operatorname{deg}(X)=n \Leftrightarrow[d, X]=n X, \quad d=N \lambda \frac{d}{d \lambda}-\operatorname{diag}[1,2, \ldots, N] .
$$

Substituting (33) into (27) and arranging in order of the degree (34), we obtain a set of equations for $u_{j}, v_{j}, \bar{u}_{j}, \bar{v}_{j}$. To write down these equations, we prepare some additional notation:

$$
\begin{array}{ll}
\mathcal{U}=\left(\mathcal{U}^{(0)}, \mathcal{U}^{(1)}, \ldots, \mathcal{U}^{(N-1)}\right), & \mathcal{V}=\left(\mathcal{V}^{(0)}, \mathcal{V}^{(1)}, \ldots, \mathcal{V}^{(N-1)}\right), \\
\overline{\mathcal{U}}=\left(\overline{\mathcal{U}}^{(0)}, \overline{\mathcal{U}}^{(1)}, \ldots, \overline{\mathcal{U}}^{(N-1)}\right), & \overline{\mathcal{V}}=\left(\overline{\mathcal{V}}^{(0)}, \overline{\mathcal{V}}^{(1)}, \ldots, \overline{\mathcal{V}}^{(N-1)}\right),
\end{array}
$$

where $\overline{\mathcal{U}}^{(j)}=\mathcal{U}^{(j)}(0,1), \overline{\mathcal{V}}^{(j)}=\mathcal{V}^{(j)}(1,0)$. We denote by $h_{k}(\overline{\mathcal{U}}, \mathcal{V}) \Lambda^{k}$ the degree $k$ terms in $\left(\operatorname{det} \overline{\mathcal{U}}_{1}\right)\left(\operatorname{det} \mathcal{V}_{1}\right) \overline{\mathcal{U}}_{1}^{-1} \overline{\mathcal{U}}_{2} \mathcal{V}_{1}^{-1} \mathcal{V}_{2}$, i.e.,

$$
\left(\operatorname{det} \overline{\mathcal{U}}_{1}\right)\left(\operatorname{det} \mathcal{V}_{1}\right) \overline{\mathcal{U}}_{1}^{-1} \overline{\mathcal{U}}_{2} \mathcal{V}_{1}^{-1} \mathcal{V}_{2}=\sum_{k=0}^{2 N} h_{k}(\overline{\mathcal{U}}, \mathcal{V}) \Lambda^{k}
$$

For example, $h_{0}(\overline{\mathcal{U}}, \mathcal{V}), h_{1}(\overline{\mathcal{U}}, \mathcal{V})$ are given by

$$
\begin{aligned}
& h_{0}(\overline{\mathcal{U}}, \mathcal{V})=\overline{\mathcal{U}}^{(0)} \mathcal{V}^{(0)} \\
& h_{1}(\overline{\mathcal{U}}, \mathcal{V})=\overline{\mathcal{U}}^{(0)}\left(\mathcal{I}-\mathcal{V}^{(0)} \mathcal{V}^{(1)}\right)+\left(\mathcal{I}-\overline{\mathcal{U}}^{(0)} \overline{\mathcal{U}}^{(1)}\right) \mathcal{V}^{(1)}
\end{aligned}
$$

By using this notation, the discrete Lax equation (27) can be rewritten as

$$
h_{k}(\overline{\mathcal{U}}, \mathcal{V})=h_{k}(\overline{\mathcal{V}}, \mathcal{U}), \quad k=0,1, \ldots, 2 N .
$$

The system of polynomial equations (35) is of course overdetermined. However, it has a unique nontrivial solution. For the moment, we consider only two equations,

$$
h_{0}(\overline{\mathcal{U}}, \mathcal{V})=h_{0}(\overline{\mathcal{V}}, \mathcal{U}), \quad h_{1}(\overline{\mathcal{U}}, \mathcal{V})=h_{1}(\overline{\mathcal{V}}, \mathcal{U})
$$

To solve (36), we introduce another set of variables $[5,18]$ :

$$
x_{j}=\prod_{i=0}^{j} v_{k}, \quad \bar{x}_{j}=\prod_{i=0}^{j} \bar{v}_{k}, \quad j=0,1, \ldots, N-2 .
$$

It is straightforward to show that $\vec{x}={ }^{t}\left[1: x_{0}: \cdots: x_{N-2}\right] \in \mathbb{P}^{N-1}$ satisfies a linear equation,

$$
\begin{aligned}
& M \vec{x}=\overrightarrow{0}, \\
& M=\left[\begin{array}{cccccc}
-u_{0} \bar{v}_{0} p_{1} & q_{0} & -p_{0} & & & \\
& -u_{1} \bar{v}_{1} p_{2} & q_{1} & -p_{1} & & \\
& & \ddots & \ddots & \ddots & \\
& & & -u_{N-3} \bar{v}_{N-3} p_{N-2} & q_{N-3} & -p_{N-3} \\
-p_{N-2} V & & & & -u_{N-2} \bar{v}_{N-2} p_{N-1} & q_{N-2} \\
q_{N-1} V & -p_{N-1} V & & & & -u_{N-1} \bar{v}_{N-1} p_{0}
\end{array}\right]
\end{aligned}
$$

where $p_{j}=u_{j} \bar{v}_{j}-1, q_{j}=p_{j} u_{j+1}+\bar{v}_{j} p_{j+1}(j=0,1, \ldots, N-1)$, and $V=\prod_{k=0}^{N-1} v_{k}=\prod_{k=0}^{N-1} \bar{v}_{k}$. 
Theorem 1. Assume that

$$
u_{j} \bar{v}_{j} \neq 1, \quad \sum_{k=0}^{n}\left\{\prod_{l=0}^{k-1} \bar{v}_{j+l} \cdot\left(u_{j+k} \bar{v}_{j+k}-1\right) \cdot \prod_{l=k+1}^{n} u_{j+l}\right\} \neq 0, \quad j=0,1, \ldots, N-1 .
$$

Under this assumption, the space of solutions for the linear equation (37) is one-dimensional, and $a$ basis is given by

$$
{ }^{t}\left[1: x_{0}: x_{1}: \cdots: x_{N-2}\right]={ }^{t}\left[g_{0}: \bar{v}_{0} g_{1}: \bar{v}_{0} \bar{v}_{1} g_{2}: \cdots:\left(\bar{v}_{0} \cdots \bar{v}_{N-2}\right) g_{N-1}\right],
$$

where $g_{j}=g_{j}(\boldsymbol{u}, \overline{\boldsymbol{v}})(j=0,1, \ldots, N-1)$ are defined as $(29)$.

To prove this theorem, we prepare two lemmas.

Lemma 1. Let $D_{n}(m)$ be the determinant of order $n$ :

$$
D_{n}(m)=\left|\begin{array}{cccc}
q_{m} & -p_{m} & & \\
-u_{m+1} \bar{v}_{m+1} p_{m+2} & q_{m+1} & \ddots & \\
& \ddots & \ddots & -p_{m+n-2} \\
& & -u_{m+n-1} \bar{v}_{m+n-1} p_{m+n} & q_{m+n-1}
\end{array}\right| .
$$

Then $D_{n}(m)$ can be expressed as

$$
D_{n}(m)=\prod_{i=1}^{n-1}\left(u_{m+i} \bar{v}_{m+i}-1\right) \cdot \sum_{j=0}^{n}\left\{\prod_{k=0}^{j-1} \bar{v}_{m+k} \cdot\left(u_{m+j} \bar{v}_{m+j}-1\right) \cdot \prod_{k=j+1}^{n} u_{m+k}\right\} .
$$

Proof. It is enough to prove the case $m=0$. Using the recurrence relation $D_{n+2}(0)=$ $q_{n+1} D_{n+1}(0)-u_{n+1} \bar{v}_{n+1} p_{n} p_{n+2} D_{n}(0)$, one can prove the Lemma 1 by induction on $n$.

Lemma 2. $\operatorname{det} M=0$.

Proof. The desired result follows from (40) and a judicious expansion of $M$ involving the $(N-1)$-th and $N$-th rows.

Proof of Theorem 1. Denote by $M_{N}^{1}$ the matrix that results from $M$ by deleting the $N$-th row and the first column. Under the assumption (38), $\operatorname{det} M_{N}^{1}=D_{N-1}(0) \neq 0$. Together with $\operatorname{det} M=0$ (Lemma 2), it follows that $\operatorname{dim} \operatorname{Ker} M=1$. The solution (39) can be checked by direct substitution into (37).

Thus we have obtained (29). Next we will show that every relation in (35) holds if the variables $\boldsymbol{u}, \boldsymbol{v}, \overline{\boldsymbol{u}}, \overline{\boldsymbol{v}}$ satisfy the relations (29).

For $\boldsymbol{a}=\left(a_{0}, \ldots, a_{N-1}\right), \boldsymbol{b}=\left(b_{0}, \ldots, b_{N-1}\right)$, we define polynomials $g_{j}^{(m)}(\boldsymbol{a}, \boldsymbol{b})(j, m=0,1, \ldots$, $N-1)$ as

$$
g_{j}^{(m)}(\boldsymbol{a}, \boldsymbol{b})=\sum_{k=0}^{m}\left\{\prod_{l=0}^{k-1} a_{j+l} \cdot\left(a_{j+k} b_{j+k}-1\right) \cdot \prod_{l=k+1}^{m} b_{j+l}\right\} .
$$

Lemma 3. If the variables $\boldsymbol{u}, \boldsymbol{v}, \overline{\boldsymbol{u}}, \overline{\boldsymbol{v}}$ obey the relation (29), then the following relations hold:

$$
g_{j}^{(m)}(\overline{\boldsymbol{u}}, \boldsymbol{v})=g_{j}^{(m)}(\overline{\boldsymbol{v}}, \boldsymbol{u}), \quad j, m=0,1, \ldots, N-1 .
$$


Proof. Since the equation is invariant under the rotation of the suffices, i.e. $0 \mapsto 1 \mapsto 2 \mapsto$ $\cdots \mapsto N-1 \mapsto 0$, one can set $j=0$ without loss of generality. The desired result can be proved by induction on $m$, using the recurrence relation

$$
g_{0}^{(m+1)}(\boldsymbol{a}, \boldsymbol{b})=g_{0}^{(m)}(\boldsymbol{a}, \boldsymbol{b}) b_{m}+\left(a_{0} \cdots a_{m-1}\right)\left(a_{m} b_{m}-1\right) .
$$

A straightforward calculation shows that

$$
\begin{aligned}
& h_{0}(\overline{\mathcal{U}}, \mathcal{V})=g_{0}^{(0)}(\overline{\mathcal{U}}, \mathcal{V})+\mathcal{I} \\
& h_{1}(\overline{\mathcal{U}}, \mathcal{V})=-g_{0}^{(1)}(\overline{\mathcal{U}}, \mathcal{V}) \\
& h_{j}(\overline{\mathcal{U}}, \mathcal{V})=(-1)^{j}\left\{g_{0}^{(j)}(\overline{\mathcal{U}}, \mathcal{V})+g_{0}^{(j-2)}(\overline{\mathcal{U}}, \mathcal{V})\right\}, \quad 2 \leq j \leq N-1, \\
& h_{N}(\overline{\mathcal{U}}, \mathcal{V})=(-1)^{N}\left\{\overline{\mathcal{U}}^{(0)} \mathcal{V}^{(0)} g_{1}^{(N-2)}(\overline{\mathcal{U}}, \mathcal{V})-g_{0}^{(N-2)}(\overline{\mathcal{U}}, \mathcal{V})-\prod_{i=0}^{N-1} \overline{\mathcal{U}}^{(i)}-\prod_{i=0}^{N-1} \mathcal{V}^{(i)}\right\}, \\
& h_{2 N-k}(\overline{\mathcal{U}}, \mathcal{V})=(-1)^{k} \prod_{i=0}^{N-k}\left(\overline{\mathcal{U}}^{(i)} \mathcal{V}^{(i)}\right) g_{N-k+1}^{(k-2)}(\overline{\mathcal{U}}, \mathcal{V}) \\
& \quad+(-1)^{k+1} \prod_{i=0}^{N-k-2}\left(\overline{\mathcal{U}}^{(i)} \mathcal{V}^{(i)}\right) g_{N-k-1}^{(k)}(\overline{\mathcal{U}}, \mathcal{V}) \quad(2 \leq k \leq N-1), \\
& h_{2 N-1}(\overline{\mathcal{U}}, \mathcal{V})=\prod_{i=0}^{N-3}\left(\overline{\mathcal{U}}^{(i)} \mathcal{V}^{(i)}\right) g_{N-2}^{(1)}(\overline{\mathcal{U}}, \mathcal{V}), \\
& h_{2 N}(\overline{\mathcal{U}}, \mathcal{V})=\prod_{i=0}^{N-2}\left(\overline{\mathcal{U}}^{(i)} \mathcal{V}^{(i)}\right) .
\end{aligned}
$$

Lemma 3 and relation (41) imply that the overdetermined system (35) is satisfied if the variables $\boldsymbol{u}, \boldsymbol{v}, \overline{\boldsymbol{u}}, \overline{\boldsymbol{v}}$ obey the relation (29). In other words, this means that the discrete Lax equation (27) is equivalent to (29).

Just as the Yang-Baxter map (29) was shown to be equivalent to (36), under the condition (38), the solitonic map (28) can also be shown to be equivalent to (36). We omit however the details of this proof.

\section{Acknowledgments}

The authors are grateful to Professors Atsuo Kuniba, Masato Okado, and Yasuhiko Yamada for discussions and comments. S.K. wishes to acknowledge support from the Japan Society for the Promotion of Science (JSPS) through a Grant-In-Aid for Scientific Research (No. 19540228); R.W. also acknowledges support by JSPS through a Grant-in-Aid (No. 21540210) and J.J.C.N. and R.W. acknowledge financial support from the British Council (PMI2 Research Co-operation award).

\section{References}

[1] Adler V.E., Bobenko A.I., Suris Yu B., Classification of integrable equations on quad-graphs. The consistency approach, Comm. Math. Phys. 233 (2003), 513-543, nlin.SI/0202024.

[2] Date E., Jimbo M., Kashiwara M., Miwa T., Transformation groups for soliton equations. Euclidean Lie algebras and reduction of the KP hierarchy, Publ. Res. Inst. Math. Sci. 18 (1982), 1077-1110.

[3] Date E., Jimbo M., Miwa T., Method for generating discrete soliton equations. II, J. Phys. Soc. Japan 51 (1982), 4125-4131. 
[4] Date E., Jimbo M., Miwa T., Method for generating discrete soliton equations. V, J. Phys. Soc. Japan 52 (1983), 766-771.

[5] Etingof P., Geometric crystals and set-theoretical solutions to the quantum Yang-Baxter equation, Comm. Algebra 31 (2003), 1961-1973, math.QA/0112278.

[6] Hatayama G., Hikami K., Inoue R., Kuniba A., Takagi T., Tokihiro T., The $A_{M}^{(1)}$ automata related to crystals of symmetric tensors, J. Math. Phys. 42 (2001), 274-308, math.QA/9912209.

[7] Hatayama G., Kuniba A., Takagi T., Soliton cellular automata associated with crystal bases, Nuclear Phys. B 577 (2000), 619-645, solv-int/9907020.

[8] Hirota R., Nonlinear partial difference equations. I. A difference analogue of the Korteweg-de Vries equation, J. Phys. Soc. Japan 43 (1977), 1424-1433.

[9] Hirota R., Discrete analogue of a generalized Toda equation, J. Phys. Soc. Japan 50 (1981), 3785-3791.

[10] Hirota R., Ultradiscretization of the Sawada-Kotera equation, in Mathematics and Physics in Nonlinear Waves (November 6-8, 2008, Fukuoka, Japan), Reports of RIAM Symposium, Vol. 20ME-S7, Research Institute for Applied Mechanics, Kyushu University, 2009, 76-85 (in Japanese).

[11] Kakei S., Nimmo J.J.C., Willox R., Yang-Baxter maps and the discrete KP hierarchy, Glasg. Math. J. 51 (2009), no. A, 107-119.

[12] Maillet J.M., Nijhoff F.W., Integrability for multidimensional lattice models, Phys. Lett. B 224 (1989), 389-396.

[13] Miwa T., On Hirota's difference equations, Proc. Japan Acad. Ser. A Math. Sci. 58 (1982), 9-12.

[14] Nimmo J.J.C., Darboux transformations and the discrete KP equation, J. Phys. A: Math. Gen. 30 (1997), 8693-8704.

[15] Nimmo J.J.C., Darboux transformations for discrete systems, Chaos Solitons Fractals 11 (2000), 115-120.

[16] Papageorgiou V.G., Suris Yu.B., Tongas A.G., Veselov A.P., On quadrirational Yang-Baxter maps, arXiv:0911.2895.

[17] Papageorgiou V.G., Tongas A.G., Veselov A.P., Yang-Baxter maps and symmetries of integrable equations on quad-graphs, J. Math. Phys. 47 (2006), 083502, 16 pages, math.QA/0605206.

[18] Suris Yu.B., Veselov A.P., Lax matrices for Yang-Baxter maps, J. Nonlinear Math. Phys. 10 (2003), suppl. 2, 223-230, math.QA/0304122.

[19] Takagi T., Soliton cellular automata, in Combinatorial Aspect of Integrable Systems, MSJ Mem., Vol. 17, Math. Soc. Japan, Tokyo, 2007, 105-144.

[20] Takahashi D., Matsukidaira J., Box and ball system with a carrier and ultradiscrete modified KdV equation, J. Phys. A: Math. Gen. 30 (1997), L733-L739.

[21] Takahashi D., Satsuma J., A soliton cellular automaton, J. Phys. Soc. Japan 59 (1990), 3514-3519.

[22] Tokihiro T., Takahashi D., Matsukidaira J., Satsuma J., From soliton equations to integrable cellular automata through a limiting procedure, Phys. Rev. Lett. 76 (1996), 3247-3250.

[23] Veselov A.P., Yang-Baxter maps and integrable dynamics, Phys. Lett. A 314 (2003), 214-221, math.QA/0205335.

[24] Veselov A.P., Yang-Baxter maps: dynamical point of view, in Combinatorial Aspect of Integrable Systems, MSJ Mem., Vol. 17, Math. Soc. Japan, Tokyo, 2007, 145-167, math.QA/0612814.

[25] Willox R., Tokihiro T., Satsuma J., Darboux and binary Darboux transformations for the nonautonomous discrete KP equation, J. Math. Phys. 38 (1997), 6455-6469.

[26] Willox R., Tokihiro T., Satsuma J., Nonautonomous discrete integrable systems, Chaos Solitons Fractals 11 (2000), 121-135. 